\title{
Perfil de Consumo de Substâncias Psicoativas por Adolescentes Escolares do Ensino Fundamental da Grande Porto Alegre/RS
}

\author{
Alexandre Dido Balbinot ${ }^{* a}$, Gabriel Soares Ledur Alves ${ }^{b} \&$ Renata Brasil Araujo $^{c}$ \\ ${ }^{a}$ Universidade Federal do Rio Grande do Sul, Porto Alegre, Brasil, ${ }^{b}$ Hospital Psiquiátrico São Pedro, Porto Alegre, Brasil \& \\ ${ }^{\mathrm{c}}$ Pontifícia Universidade Católica do Rio Grande do Sul, Porto Alegre, Brasil
}

\begin{abstract}
RESUMO
Este trabalho objetivou avaliar o perfil de consumo de substâncias psicoativas por escolares da Grande Porto Alegre/RS. Método: Estudo transversal com amostra composta por adolescentes de ambos os sexos $(\mathrm{n}=147)$. Para coleta dos dados utilizou-se questionário elaborado para tal. Resultados: A experimentação do álcool ocorreu em média aos 11,48 anos $(\mathrm{DP}=1,82)$. As substâncias mais utilizadas na vida foram: álcool por $80 \%(\mathrm{DP}=54,4)$ da amostra, tabaco por $14 \%(\mathrm{DP}=9,5)$ e maconha por 3\% $(\mathrm{DP}=2,0)$. Evidenciou-se associação entre percepção sobre problemas com álcool e: problemas com tabaco $(\mathrm{p}<0,001)$, com maconha $(\mathrm{p}=0,041)$ e com anabolizantes $(\mathrm{p}=0,008)$. Conclusão: A amostra apresentou uso precoce de álcool e associação entre a percepção de problemas decorrentes do álcool e problemas com: tabaco, maconha e anabolizantes.
\end{abstract}

Palavras-chave: adolescentes; drogadição; substâncias psicoativas.

\section{ABSTRACT \\ Profile of Consumption of Psychoactive Substances in Teens Students of Elementary School From The Great Porto Alegre/RS}

This study aimed to evalute the profile of psychoactive substances consumption by students of Porto Alegre/RS. Method: Cross-sectional study with a sample of adolescents of both sexes $(n=147)$. For data collection we used questionnaire prepared for that. Results: The trial of alcohol occurred on average at 11.48 years $(\mathrm{SD}=1.82)$. The substance most commonly used in life were: alcohol by $80 \%$ $(\mathrm{SD}=54.4)$ of the sample, tobacco by $14 \%(\mathrm{SD}=9.5)$ and cannabis by $3 \%(\mathrm{SD}=2.0)$. Associations were observed between the perceptions about problems with alcohol and: problems with tobacco use ( $\mathrm{p}<0.001)$, problems with marijuana $(\mathrm{p}=0.041)$ and problems with steroids $(\mathrm{p}=0.008)$. Conclusion: The sample showed early alcohol use and association between the perception of problems related to alcohol and have problems with: tobacco, marijuana and anabolic steroids.

Keywords: adolescents; drug addiction; psychoactive substances.

A adolescência é uma fase do desenvolvimento que vem acompanhada de muitas mudanças biológicas, cognitivas, emocionais e sociais, que fazem com que os indivíduos que passam por ela, estejam mais expostos a comportamentos de risco à saúde, como: níveis insuficientes de atividade física, consumo de drogas lícitas e ilícitas e hábitos alimentares inadequados (Farias Junior, 2008; Vieira, Aerts, Freddo, \& Monteiro, 2008;).

Rappaport (1995) relata ser comum, no período da adolescência, o jovem relatar a necessidade de pertencer a um grupo, aspecto que demonstra a representati- vidade dos amigos como figuras influenciadoras, e deste modo, importantes nesse período. Nesta fase, os adolescentes apresentam como características a contestação e a curiosidade, portanto, mais sujeitos aos comportamentos de risco. Segundo Robaina (2010), os adolescentes iniciam o consumo de drogas por curiosidade, como uma forma de desafio ou para fugir da realidade em que vivem. A facilidade de acesso às substâncias é destacada pelo autor como um fator que aumenta a probabilidade da ocorrência deste comportamento, já que nos ambientes que costumam ser frequentados pelos jovens, como escolas, bares e boates, as drogas podem ser obtidas sem grandes dificuldades.

Endereço para correspondência: Alexandre Dido Balbinot - adbalbinot@gmail.com 
$\mathrm{Na}$ adolescência, frequentemente, ocorre a experimentação de substâncias psicoativas, as quais, para grande parte dos adolescentes será apenas parte de seu processo de desenvolvimento, cessando-se tão rapidamente quanto seja sanada sua momentânea necessidade de uso. Todavia, alguns desenvolverão um uso prolongado, crônico e problemático, resultando em um prejuízo para a própria vida e para a dos indivíduos ao seu entorno, muitas vezes chegando à dependência e necessitando a utilização de diversos dispositivos de tratamento para adquirir a condição de autonomia e retomada da qualidade de vida que apresentavam anteriormente. Esta utilização das drogas lícitas ou ilícitas está inserida na cultura da adolescência à velhice e, no caso do Brasil, podemos observar que ela se inicia a partir do consumo de álcool, tabaco e maconha (Schenker \& Minayo, 2005; Tavares, Béria, \& Lima, 2004).

De acordo com o "V Levantamento nacional sobre o consumo de drogas psicotrópicas entre estudantes do ensino fundamental e médio da rede pública de ensino nas 27 capitais brasileiras", o consumo de substâncias psicoativas começa antes mesmo da adolescência. $\mathrm{O}$ estudo explicita também que as drogas lícitas (tabaco e álcool) são aquelas consideradas as de primeiro uso na vida. O álcool é uma das principais drogas lícitas consumidas na adolescência, sendo comum também a busca por novas experiências nesta fase do desenvolvimento, aliada a comportamentos de impulsividade, ansiedade, insegurança, insatisfação e agressividade (Chambers, Taylor, \& Potenza, 2003). Neste sentido, o uso de álcool preenche todos os requisitos para complementar uma adolescência em desarmonia: prazer imediato, transgressão, necessidade de liberdade, aceitação e respeito com colegas/amigos (Cavaiola \& Schiff, 1989).

Bucher (1992) colabora salientando que os jovens iniciam suas experiências com as drogas consideradas lícitas em seus ambientes familiares, podendo após recorrer às ilícitas para aumentar o nível de prazer decorrente do uso, procurando outras emoções ou fugir de seus problemas. Segundo Noto, Galduróz, Nappo e Carlini (2004), substâncias psicoativas ilícitas são utilizadas pela primeira vez após a experimentação ou uso das drogas lícitas.

Além das informações supracitadas, estudos demonstram que adolescentes não associam o uso de substâncias psicoativas aos riscos dele decorrentes. Entretanto, Barreto, Giatti, Casado, Moura, Crespo e Malta (2010) encontraram associação positiva entre tabagismo e outros comportamentos de risco para a saúde. Outros achados mostram que em grande parte os adolescentes conseguem realizar as atividades de seu cotidiano (como a prática de esportes e o trabalho) apesar do uso de substâncias psicoativas (Eitle \& Eitle, 2004; Peretti-Watel \& Lorente, 2004).

Cabe salientar, que fatores de risco para uso de drogas entre adolescentes no Brasil têm sido pouco estudados, sendo a maior parte das informações disponíveis a esse respeito proveniente de estudos realizados em outros países (Tavares, Béria, \& Lima, 2004). Assim, o presente trabalho tem por objetivo avaliar o perfil de consumo de substâncias psicoativas por escolares pertencentes à Grande Porto Alegre/RS.

\section{MÉTODO}

Trata-se de uma pesquisa com delineamento transversal. A amostra é composta por 147 adolescentes, com idades entre 12 e 17 anos, de ambos os sexos, pertencentes a duas escolas públicas de ensino fundamental, sendo ambas da região metropolitana de Porto Alegre-RS. Assim como a escolha das escolas, as turmas foram selecionadas por conveniência, sendo, as coletas, realizadas em todas as turmas de $5^{\mathrm{a}}, 6^{\mathrm{a}}, 7^{\mathrm{a}} \mathrm{e}$ $8^{\mathrm{a}}$ séries do ensino fundamental.

O cálculo do tamanho da amostra foi realizado utilizando-se para isto a análise dos dados do "V levantamento nacional sobre o consumo de drogas psicotrópicas entre estudantes do ensino fundamental e médio da rede pública de ensino nas 27 capitais brasileiras" (Noto, Galduróz, Nappo, \& Carlini, 2004). Foi possível estimar um número de 147 participantes, tendo $90 \%$ de confiança e $6.7 \%$ de erro. Utilizou-se para isto, a seguinte fórmula:

$$
n=\frac{N \cdot Z^{2} \cdot p \cdot(1-p)}{Z^{2} \cdot p \cdot(1-p)+e^{2} \cdot(N-1)}
$$

Sendo:

$\mathrm{n}$ - amostra calculada

$\mathrm{N}$ - população de estudantes

$\mathrm{Z}$ - variável normal padronizada associada ao nível de confiança

$\mathrm{p}$ - verdadeira probabilidade do evento

e - erro amostral

Os critérios de exclusão da amostra foram: ter diagnóstico prévio de retardo mental com laudo; ser deficiente visual; ter alguma alteração que prejudicasse o preenchimento dos instrumentos no momento da aplicação.

Não foram realizadas exclusões de sujeitos da amostra devido aos critérios preestabelecidos. 


\section{Instrumentos}

Ficha de dados sociodemográficos e de avaliação do uso de substâncias psicoativas. Elaborada para definir o perfil sociodemográfico da amostra estudada, identificando características referentes ao padrão de consumo, e demais aspectos importantes para melhor compreensão do indivíduo e de seu uso de substâncias psicoativas.

\section{Aspectos Éticos}

O presente estudo foi aprovado pelo Comitê de Ética em Pesquisa do Hospital Psiquiátrico São Pedro. Posteriormente, para realização da pesquisa, houve a autorização por parte dos diretores das instituições escolares. Para a obtenção da autorização dos responsáveis pelo aluno, os pesquisadores entregaram três dias antes da coleta o termo de consentimento livre e esclarecido a cada aluno, para ser encaminhado aos pais. Nesse período, os pesquisadores ficaram disponíveis para o contato telefônico e/ou pessoal. Todos os pais concordaram com a participação de seus filhos na pesquisa.

\section{Procedimentos para a Coleta de Dados}

$\mathrm{Na}$ data da coleta dos dados, os alunos que retornaram com termo de consentimento dos responsáveis assinados foram convidados a participar do estudo. Em caso de aceite, o aluno também assinava um termo de consentimento e respondia ao questionário, em sala de aula. A aplicação foi realizada individualmente.

A aplicação do instrumento foi realizada por profissionais treinados. Os horários de coleta foram decididos juntamente com a coordenação das escolas, e a entrada na sala de aula dava-se no momento em que os alunos se encontravam em horário letivo. Para coleta, a aula era interrompida e retomada quando findado o procedimento, havendo sempre a concordância do professor que permanecia na sala apenas observando a aplicação.

\section{ANÁLISE DOS DADOS}

Os dados foram analisados através do software Statistical Package for the Social Sciences (SPSS), versão 17.0. A análise dos dados contou com testes Descritivos e de Frequências e o Teste Exato de Fisher. O nível de significância utilizada como parâmetro foi o de $5 \%$.

\section{RESULTADOS}

A amostra foi constituída por 147 indivíduos, sendo que destes, $23,1 \%(n=34)$ era proveniente de escola da cidade de Porto Alegre, enquanto 76,9\% ( $n=113)$ de escola da cidade de Gravataí. Através dos dados provenientes da amostra, foi possível observar que $44,2 \%$ eram do sexo masculino $(n=50)$, enquanto que $55,8 \%$, do sexo feminino $(n=63) ; 100 \%(n=147)$ declararam ser solteiros. A idade média dos participantes foi de 13,47 anos $(D P=1,11 ; 11-17)$. Referente à escolaridade, obteve-se uma média de 7,5 anos estudados $(\mathrm{DP}=1,10 ; 5-13)$, o que equivale ao ensino fundamental incompleto.

Em relação ao trabalho, $10,9 \%(\mathrm{n}=16)$ dos indivíduos relataram realizar trabalho remunerado; $87,8 \%$ $(n=129)$ relataram que não trabalham de forma remunerada; e 1,4\% ( $n=2)$ não responderam. Contudo, ao ser abordado o trabalho de forma geral (com ou sem remuneração $) 13,6 \% \quad(n=20)$ relataram trabalhar; $72,8 \%(n=107)$ relataram não trabalhar; e $13,6 \%(n=$ 20) não responderam. Por fim, ao abordar a quantidade de horas diárias trabalhadas, foi evidenciada uma média de 3,20 horas semanais ( $\mathrm{DP}=1,75 ; 1-5)$.

Foi questionada a saúde geral, através de cinco alternativas, sendo elas: "Excelente", "Muito Boa", "Boa", "Regular", "Ruim". Desta maneira observouse que $31,3 \%(n=46)$ responderam "Excelente"; $38,1 \%(\mathrm{n}=56)$ responderam "Muito Boa"; $21,8 \%(\mathrm{n}=$ 32) responderam "Boa"; $6,8 \%(n=10)$ responderam "Regular"; não houve respostas para a alternativa "Ruim"; e 2\% (n=3) não respondeu.

$\mathrm{Na}$ Tabela 1 é apresentado, por substância, o percentual de indivíduos que realizaram uso em pelo menos um episódio durante a vida, tão qual o percentual de indivíduos que reconheceram ter tido problemas decorrentes do uso. 
Tabela 1

Percentual de Uso de Substâncias Psicoativas e de Problemas Decorrentes do Uso ( $n=147)$

\begin{tabular}{ccc}
\hline Substância & Uso na vida $\mathrm{n}(\%)$ & Problemas decorrentes do uso $\mathrm{n}(\%)$ \\
\hline Álcool & $80(54,4)$ & $30(37,5)$ \\
Tabaco & $14(9,5)$ & $11(78,57)$ \\
Maconha & $3(2,0)$ & $2(66,67)$ \\
Alucinógenos & $1(0,7)$ & $1(100)$ \\
Sedativos e/ou Barbitúricos & $1(0,7)$ & $1(100)$ \\
Anabolizantes & $4(2,7)$ & $3(75)$ \\
Tranquilizantes & $9(6,1)$ & $2(22,22)$ \\
Anfetaminas & $9(6,1)$ & $4(44,44)$ \\
\hline
\end{tabular}

Os dados referentes a demais substâncias (solvente, cocaína ev, cocaína inalada, cocaína fumada/crack) não foram apresentados por não terem sido utilizadas pela amostra.

Já, na Tabela 2, podem ser observados: a idade de início do uso de cada substância psicoativa ao ser questionado a idade de experimentação; a quantidade média utilizada por semana no último mês, ao ser questionado a quantidade utilizada de cada substância nos últimos 30 dias; e o tempo decorrido desde o último consumo até o momento da coleta dos dados, ao ser questionado quando foi realizado pela última vez o uso (posteriormente sendo feita, pelos pesquisadores, a computação do tempo transcorrido entre este uso e a data da coleta).

Tabela 2

Padrão de Consumo de Substâncias Psicoativas

\begin{tabular}{cccc} 
Substância & Idade de início (DP) & $\begin{array}{c}\text { Quantidade média utilizada } \\
\text { por semana* (DP) }\end{array}$ & Último uso em dias (DP) \\
\hline Álcool & $11,48(1,82)$ & $2,78(2,99)$ & $66,54(193,46)$ \\
Tabaco & $13,08(1,73)$ & $5,00(6,73)$ & $165,57(403,77)$ \\
Maconha & $11,67(0,57)$ & 1,00 & 90,00 \\
Anabolizantes & $15,00(0,00)$ & $2,67(2,88)$ & $64,67(100,06)$ \\
Tranquilizantes & $12,50(1,29)$ & 1,00 & 1,00 \\
Anfetaminas & $13,83(0,75)$ & $2,50(2,12)$ & $98,00(154,94)$ \\
Alucinógenos & 13,00 & 1,00 & 240,00 \\
Sedativos elou Barbitúricos & 12,00 & - & -
\end{tabular}

Nota. *Para a mensuração da quantidade média utilizada por semana no último mês, foram utilizados os seguintes padrões de medidas: Unidades Internacionais/ UI (álcool); cigarros (tabaco); baseados (maconha); comprimidos (anabolizante, tranquilizante, medicação para emagrecer sem prescrição médica, alucinógeno e/ou barbitúrico); gramas (cocaína/ crack); litros (solvente). Uma UI de álcool equivale a 10 gramas.

De acordo com o Teste Exato de Fisher, foi encontrada associação entre a percepção do adolescente quanto a ter problemas com o álcool e ter problemas com o tabaco $(\mathrm{p}<0,001)$; ter problemas com a maco- nha $(\mathrm{p}=0,041)$; e ter problemas com anabolizantes $(\mathrm{p}=0,008)$. Não foi encontrada associação com a percepção de ter problemas com outras substâncias psicoativas. 


\section{DISCUSSÃO}

Referente ao percentual de indivíduos que já fizeram uso na vida de cada substância, quando comparado com a literatura, nota-se uma média de consumo de substâncias psicoativas abaixo do que é encontrado em estudos realizados em cidades do estado de São Paulo e do interior do Rio Grande do Sul (Guimarães, Godinho, Cruz, Kappann, \& Tosta Junior, 2004; Muza, Bettiol, Muccillo, \& Barbieri, 1997; Silva, Pavani, Moraes, \& Chiaravalloti Neto, 2006; Sengik \& Scortegagna, 2008; Silva, Pavani, Moraes, \& Chiaravalloti Neto, 2007).

Semelhantemente, em comparativo com o "V Levantamento nacional sobre o consumo de drogas psicotrópicas entre estudantes do ensino fundamental e médio da rede pública de ensino nas 27 capitais brasileiras", realizado pelo Centro Brasileiro de Informações sobre Drogas Psicotrópicas (CEBRID), mais especificamente dos dados relativos a Porto Alegre, também podemos observar um percentual menor de uso na vida em relação a todas as substâncias. Fato que pode estar associado à maior idade média da amostra presente no estudo do CEBRID (Noto, Galduróz, Nappo, \& Carlini, 2004).

Já o uso de álcool na vida se aproxima ao encontrado no "II Levantamento domiciliar sobre o uso de drogas psicotrópicas no Brasil", no qual foi encontrado um percentual de $58,8 \%$ para a faixa etária de 12 a 17 anos. A utilização, na vida, de alucinógenos, sedativos e anabolizantes tiveram percentuais maiores, enquanto que tabaco, solvente, cocaína e crack obtiveram percentuais menores que o encontrado naquele estudo (Silva, Noto, Fonseca, Carlini, Oliveira, Nappo, Moura, \& Sanchez, 2006).

No presente estudo as duas substâncias que tiveram maior percentual de uso na vida foram álcool e tabaco, semelhante ao encontrado nos estudos de Muza, Bettiol, Muccillo e Barbieri (1997) e de Silva, Pavani, Moraes e Chiaravalloti Neto (2006). Nestes dois estudos, a terceira substância mais utilizada na vida foi o solvente, diferentemente do presente estudo, no qual o terceiro lugar foi ocupado pelo uso de maconha.

Dal Pizzol, Branco, Carvalho, Pasqualotti, Maciel e Migott (2006) investigando o uso de medicamentos sem prescrição médica por adolescentes da região sul do Brasil, evidenciaram uso de ansiolíticos, anfetaminicos, anabolizantes e barbitúricos superior ao encontrado na presente pesquisa. Todavia, a diferença da idade das amostras pode ser um fator de confusão para esta comparação, explicando esta diferença.
Foi possível identificar que a ordem de progressão do consumo das substâncias psicoativas diferencia-se do que é encontrado na literatura, a qual é em indivíduos com idades entre 10 e 12 anos: álcool, tabaco, solvente, sedativo, maconha, crack, barbitúricos (Noto, Galduróz, Nappo, \& Carlini, 2004). A progressão encontrada na amostra do presente estudo foi: álcool; maconha; sedativos; tranquilizantes; alucinógenos; tabaco; medicação para emagrecer sem prescrição médica; e, por fim, anabolizantes. Esses dados demonstram uma possível mudança no padrão de progressão do uso de substâncias psicoativas, a qual pode estar associada a inúmeros fatores como questões socioculturais e disponibilidade das substâncias.

A percepção de problemas com relação ao uso do álcool pelos adolescentes foi associada com a percepção de ter problemas com o uso do tabaco, da maconha e de anabolizantes. O fato de o álcool ser "porta de entrada" para o uso de outras substâncias já havia sido descrito por Alamarse e Carvalho (2006) e demonstra a importância do uso deste não ser minimizado pela sociedade e pela família (Silva, Pavani, Moraes, \& Chiaravalloti Neto, 2006; Schenker \& Minayo, 2005). Alguns autores destacaram que a monitorização da família quanto ao uso das drogas lícitas pelos jovens, tem um papel fundamental na prevenção do uso das drogas ilícitas (Schenker \& Minayo, 2005; Tavares, Béria, \& Lima, 2004). Deve-se lembrar, como citado por Sengik e Scortegagna (2008), que não existe uso lícito de drogas por menores de idade, sendo, inclusive, criminosa a venda realizada para este tipo de clientela. Infelizmente, a fiscalização e penalização para os proprietários que cometem essa categoria de crime não parece ser muito efetiva (Robaina, 2010).

Observaram-se como limitações do presente estudo, que podem ter interferido nos resultados, o tamanho da amostra, assim como o método de seleção desta, que ocorreu por conveniência, sendo a coleta feita em apenas duas escolas públicas. Também houve prejuízo para a pesquisa por esta ser realizada em ambiente escolar, onde mesmo com a garantia de sigilo por parte dos pesquisadores, pode ter permanecido junto à amostra o sentimento de intimidação ou penalização e o consequente medo em responder com fidedignidade as perguntas expressas no instrumento aplicado.

\section{CONSIDERAÇÕES FINAIS}

O uso na vida de substâncias psicoativas teve um percentual menor do que o encontrado na grande maioria dos estudos abordando escolares e/ou adolescentes. 
Todavia esta comparação fica um tanto quanto prejudicada devido à diferença nas idades médias das amostras, sendo a idade uma variável relevante.

A amostra apresentou um padrão de progressão do consumo diferenciado do que é observado em vários estudos brasileiros com a população adolescente. Também foi evidenciada associação entre a percepção de ter problemas com o álcool e a percepção de ter problemas com o tabaco, com a maconha e com os anabolizantes.

Por fim, destaca-se a necessidade de maiores investigações acerca dos fatores que influenciam o processo de consumo das diferentes substâncias psicoativas, tanto no que se refere à idade de início do uso e à progressão do consumo, quanto ao uso combinado de diferentes substâncias. Assim, poderão ser delineados projetos de prevenção ao uso de substâncias mais efetivos e especialmente direcionados a crianças e adolescentes.

\section{REFERÊNCIAS}

Alamarse, G. M. A., \& Carvalho, M. D. B. (2006). Álcool e adolescência: O perfil de consumidores de um município do norte do Paraná. Escola Anna Nery Revista de Enfermagem, 10, 408416.

Barreto, S. M., Giatti, L., Casado, L., Moura, L., Crespo, C., \& Malta, D. C. (2010). Exposição ao tabagismo entre escolares no Brasil. Ciência Saúde Coletiva, 15, 3027-3034.

Cavaiola, A. A., \& Schif, M. (1989). Self-esteem in abused chemically dependent adolescents. Child Abuse \& Neglect, 13, 327334.

Chambers, R. A., Taylor, J. R., \& Potenza, M. N. (2003). Developmental neurocircuitry of motivation in adolescence: A critical period of addition. American Journal of Psychiatry, 160, 1041-1052.

Dal Pizzol, T. S., Branco, M. M. N., Carvalho, R. M. A., Pasqualotti, A., Maciel, E. N., \& Migott, A. M. B. (2006). Uso nãomédico de medicamentos psicoativos entre escolares do ensino fundamental e médio no Sul do Brasil. Cadernos de Saúde Pública, 22, 109-115.

Eitle, D. J., \& Eitle, T. M. (2004). School and county characteristics as predictors of school rates of drug, alcohol, and tobacco offenses. Journal of Health and Social Behavior, 45, 408-421.

Farias Junior, J. C. (2008). Associação entre prevalência de inatividade física e indicadores de condição socioeconômica em adolescentes. Revista Brasileira de Medicina do Esporte, 14, 109-114.

Guimarães, J. L., Godinho, P. H., Cruz, R., Kappann, J. I., \& Tosta Junior, L. A. (2004). Consumo de drogas psicoativas por adolescentes escolares de Assis, São Paulo. Revista Saúde Pública, 38, 130-132.
Muza, G. M., Bettiol, H., Muccillo, G., \& Barbieri, M. A. (1997). Consumo de substâncias psicoativas por adolescentes escolares de Ribeirão Preto, SP - I: Prevalência do consumo por sexo, idade e tipo de subtância. Revista Saúde Pública, 31, 21-29.

Noto, A. R., Galduróz, J. C. F., Nappo, S. A., \& Carlini, E. A. (2004). V levantamento nacional sobre o consumo de drogas psicotrópicas entre estudantes do ensino fundamental e médio da rede pública de ensino nas 27 capitais brasileiras, 2004. São Paulo: Centro Brasileiro de Informações sobre Drogas Psicotrópicas (CEBRID) - Departamento de Psicobiologia da Escola Paulista de Medicina.

Peretti-Watel, P., \& Lorente, F. O. (2004). Cannabis use, sport practice and other leisure activities at the end of adolescence. Drugs and Alcohol Dependence, 73, 251-257.

Robaina, J. V. L. (2010). Drogas: O papel do educador na prevenção ao uso de drogas. Porto Alegre: Mediação.

Schenker, M., \& Minayo, M. C. S. (2005). Fatores de risco e de proteção para o uso de drogas na adolescência. Ciência \& Saúde Coletiva, 10, 707-717.

Silva, A. A. B., Noto, A. R., Fonseca, A. M., Carlini, E. A., Oliveira, L. G., Nappo, S. A., Moura, Y. G., \& Sanchez, Z. M. (2006). II levantamento domiciliar sobre o uso de drogas psicotrópicas no Brasil: Estudo envolvendo as 108 maiores cidades do país: 2005. São Paulo: Centro Brasileiro de Informações sobre Drogas Psicotrópicas (CEBRID) - UNIFESP-Universidade Federal de São Paulo.

Silva, E. F., Pavani, R. A. B., Moraes, M. S., \& Chiaravalloti Neto, F. (2006). Prevalência do uso de drogas entre escolares do ensino médio do município de São José do Rio Preto, São Paulo, Brasil. Cadernos de Saúde Pública, 22, 1151-1158.

Silva, E. F., Pavani, R. A. B., Moraes, M. S., \& Chiaravalloti Neto, F. (2007). Caracterização do consumo de drogas ilícitas entre escolares do ensino médio do município de São José do Rio Preto, SP, Brasil. Arquivos de Ciência da Saúde, 14, 135139.

Sengik, A. S., \& Scortegagna, S. A. (2008). Consumo de drogas psicoativas em adolescentes escolares. Revista de Psicologia da Vetor Editora, 9, 73-80.

Tavares, B. F., Béria, J. U., \& Lima, M. S. (2004). Fatores associados ao uso de drogas entre adolescentes escolares. Revista Saúde Pública, 38, 787-96.

Vieira, P. C., Aerts, D. R. G. C., Freddo, S. L., \& Monteiro, L. (2008). Uso de álcool, tabaco e outras drogas por adolescentes escolares em município do Sul do Brasil. Cadernos de Saúde Pública, 24, 2487-2498.
Recebido: 01/07/2011

Última revisão: 08/08/2012 Aceito: 09/08/2012 